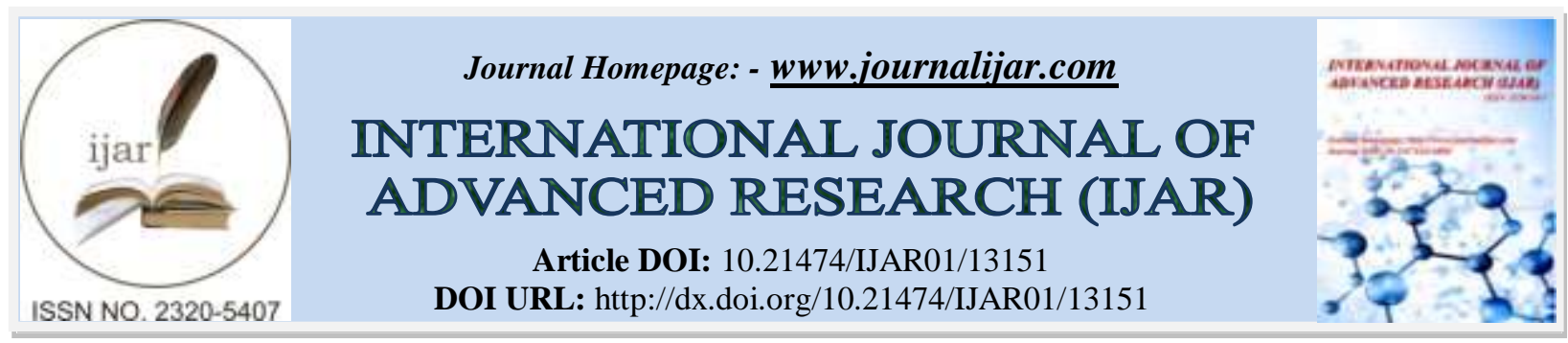

RESEARCH ARTICLE

\title{
TECHNOLOGY AND HEALTHCARE: DEVELOPMENT OF ROBOTIC SURGERY AND ITS APPLICATION
}

Thame Mcfadden and Mersey Mcfadden

Mahidol University International Demonstration School.

\section{Manuscript Info}

(.........................

Manuscript History

Received: 15 May 2021

Final Accepted: 18 June 2021

Published: July 2021

Key words:-

Da Vinci, Surgery, Robots

\begin{abstract}
Modern technology has changed our daily lives in many ways such as communication, transportation, and especially in terms of medication. Such medical technologies include robotic surgery. Robotic surgery, considered as new technology as the first one, PUMA 560, was first introduced in 1985 for stereotaxic operation- inserting a needle into the brain for biopsy. Robotic surgery helped surgeons to perform complex tasks and subjects to minimize trauma errors that might occur due to imprecision. The implications of robots are still in the early stages and there are many concerns with the use of robots in the medical field, especially in surgery. Such problems are but are not limited to safety, ethics and morals, and its ability compared to humans. In this paper, we will begin with the history and general use of robot both in daily life and the medical field and we will explore laparoscopic surgery before we deeply elaborate on the use of robots, Da Vinci (the first robotic surgery approved by the Food and Drug Administration for laparoscopic surgery). Then we will illustrate the highlighted case performed by robotic surgery and compare between robots. Last but not least, we will analyze whether robots might replace surgeons in the future and how they might impact us both positively and negatively.
\end{abstract}

Copy Right, IJAR, 2021,. All rights reserved.

\section{Introduction:-}

A robot refers to a machine that can perform tasks according to instructions. In which input information must be provided in order to perform physical actions such as walking, lifting, and moving objects. Robots have been developed for over many centuries for multi-purposes. For example, in the Before Christian Age, a robot named "The Maidservant of Philon" was created for pouring wine(Jimenez, 2014). The first robot in history that were able to think like the way the biological brain does, Elmer and Elsie, was introduced by William Grey Walter in 1948(Inglish-Arkell, 2012). They also were programmed to find charging stations once their batteries are running low(Bellis, 2019). The first industrial robot invented in 1954 by George Devol named "Unimate". After that, factories began to use robots to replace humans or work that humans cannot do(Inventor of the Robot arm and Its Continued Development, 2019). In addition to the industrial field, robots are being produced for many objectives such as construction, health, household, working with toxic substances that are dangerous for humans.

Corresponding Author:- Thame Mcfadden

Address:- Mahidol University International Demonstration School. 


\section{Laparoscopic surgery:}

Laparoscopic surgery is an operation that is performed by penetrating a very small hole through the abdominal wall. The surgeon uses a laparoscope, a long thin tube with a high-intensity light and a high-resolution camera located at the front, inserted through a penetrated abdominal wall.

The surgeon will view the image of internal organs and cavities through the TV screen while performing the surgery(Laparoscopy (keyhole surgery), 2018).

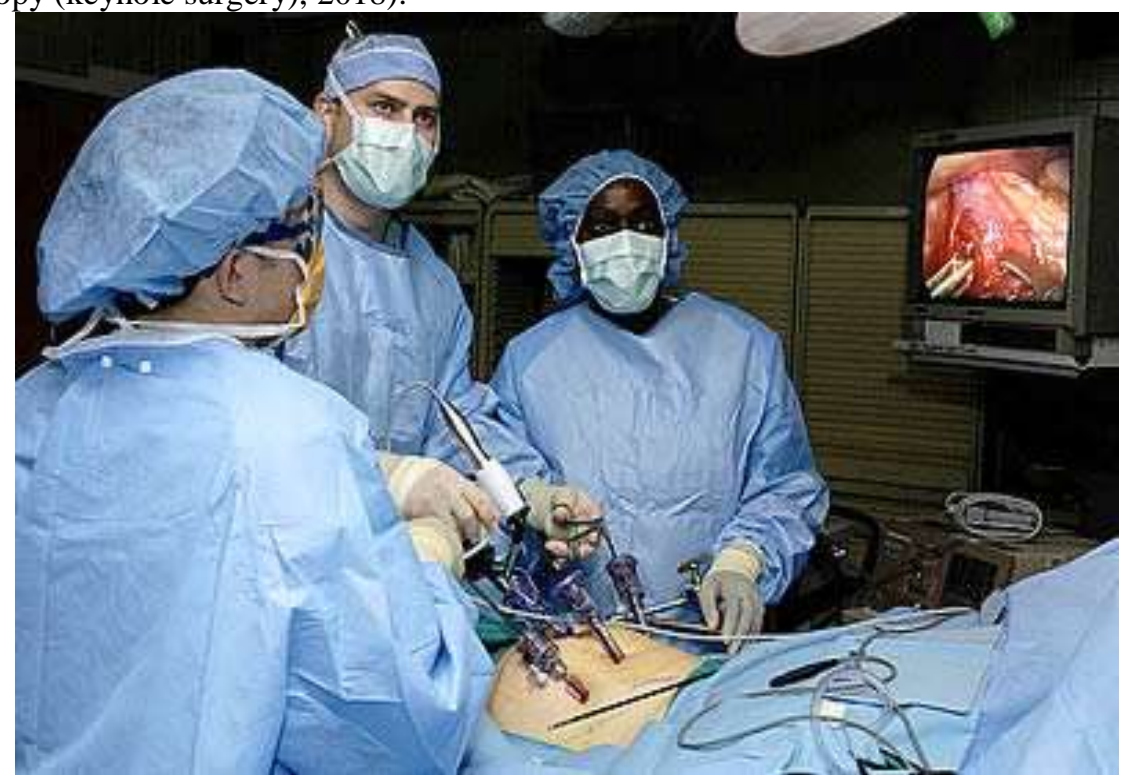

Figure 1:- Laparoscopic surgery.

Laparoscopy is used commonly to identify and diagnose many conditions such as pelvic or abdominal pain. In many cases, it is possible to diagnose conditions using non-invasive methods like ultrasound scan, computerised tomography (CT) scan, or magnetic resonance imaging (MRI) scan. Laparoscopy is performed when these tests do not provide enough information for diagnosis. Such cases may include pelvic inflammatory disease (PID); which is an infection of the female reproductive organs: womb, fallopian tubes, and ovaries. Severe pelvic inflammatory disease might cause scar tissue and painful collection of pus to develop in the female reproductive organs and lead to permanent reproductive damage such as ectopic pregnancy, infertility, chronic pelvic pain, and tubo-ovarian abscess(Pelvic inflammatory disease(PID)- Symptoms and causes, n.d.). According to Journal of obstetrics and gynaecology of India, 577 laparoscopic examinations were performed in women with sterility, chronic abdominal pain, amenorrhea, and unstable menstrual periods and 125 cases were detected as a chronic pelvic inflammatory disease both tuberculous and nonspecific type. From those 125 cases, 59 were identified as tuberculous. Further investigation into laparoscopy had yielded a result of 12 cases. 87 cases out of the 125 had no abnormal clinical findings and about 18 out of the 37 tubo-ovarian masses were diagnosed by bimanual examination. These results suggest that laparoscopy is a critical tool for diagnosis of pelvic inflammatory disease. It can reveal peritubal adhesions, tubercles on the tubes, small tubo-ovarian masses, and hydrosalpinx cases, a woman's fallopian tube blocked with fluid, that cannot be found clinically. Compared to culdoscopy, it is more suitable for PID diagnosis with more frequent adhesions to the fallopian tubes and pathological conditions in the upper pelvis. Laparoscopy can portray a role as a prelude to tuboplasty. Out of 316 cases of primary sterility examined, 51 women had a genital tuberculosis and 39 cases had nonspecific PID. Laparoscopy can be used to ensure whether the case is labelled as chronic pelvic disease is correct or not. From 15 women who reported chronic lower abdominal pain was detected as false. Only 13 cases of PID were confirmed among 33 cases detected as PID prior. However, there are possibilities of suddenly intense infection during laparoscopy that might occur(Krishna et al., 1979). robotic assisted laparoscopic surgery vs. laparoscopic surgery and open surgery

Robots allowed surgeons to perform difficult tasks with higher accuracy especially in the field of urology. There was a comparative effectiveness study done to evaluate the outcome of robotic assisted laparoscopic surgery vs. laparoscopic surgery and open surgery. The cost and inpatient outcomes were compared during this study. Robotic assisted laparoscopic performed radical prostatectomies for $52.7 \%, 27.3 \%$ for pyeloplasty, $11.5 \%$ for partial nephrectomies, and $2.3 \%$ for nephrectomies. The study suggested that robotic assisted laparoscopic surgery was 
more prevalent compared to open surgery among white patients in radical prostatectomy. Robot assisted laparoscopic surgery and laparoscopic surgery are associated with lower death rate, shorter length of stay, complications, transfusions, and more routine discharge compared to open surgery in all procedures(Hua-yin et al., 2012).

\section{Da Vinci}

Robotic- assisted surgery (Fig 2) named Da Vinci was approved by the Food and Drug Administration in 2000. Its name came from Leonardo da Vinci's study of human anatomy(Hurley, 2017).By the year of 2012, approximately 367,000 cases of surgery was performed via robotic-assisted da Vinci surgery; use of robotic technology to perform surgery under the supervision of a surgeon(Tanner, 2013).The main component of Da Vinci surgical system are:

1. Surgeon console : where the surgeon operates seated, designed according to ergonomics to suit the surgery, and supervises the surgery through a 3D image.

2. Patient-side cart: where the patient is positioned during the surgery.

3. Endowrist instruments : the tool can be rotated in seven directions, which is greater than the human wrist and a conventional laparoscopic instrument that has only 4 degrees of motion, thus endowrist instruments enable more movement than a human's hand with more accuration.

4. Vision system : 3D endoscope, flexible tube with a camera and a light at the tip, and image processing equipment that provide a true-life image quality of the patient's anatomy.(da Vinci Surgery Community, n.d.)

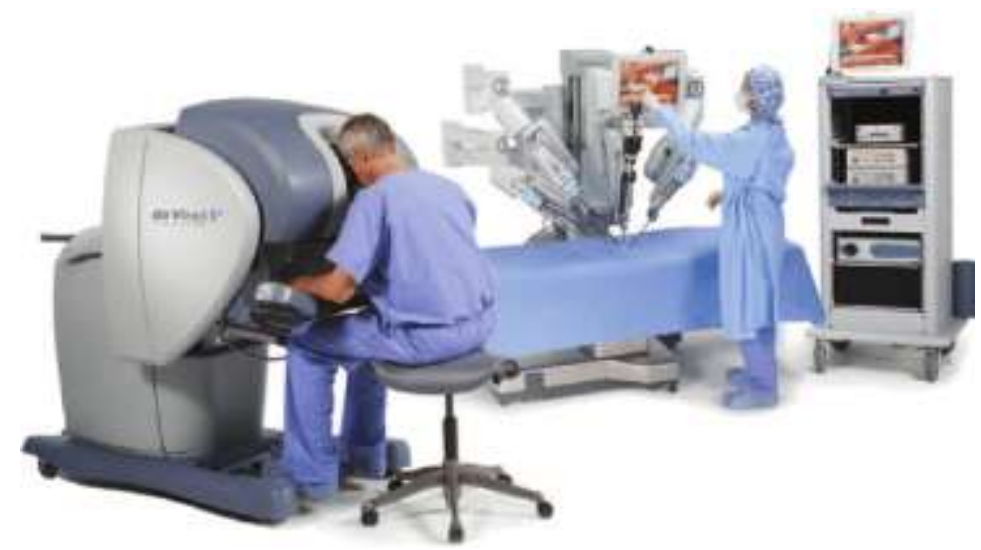

Figure 2:- Comparing da Vinci with other robot.

There was a trial of different robots performing nerve-sparing (a type of surgery that attempts to save the nerves near the tissues being removed (Medical Dictionary, n.d)) robot-assisted radical cystoprostatectomy and urinary diversion. The result illustrates that da Vinci system allows more precision and less time required to remove the bladder with less blood loss, decreased dramatically from $910 \mathrm{~mL}$ to $153 \mathrm{~mL}$, for nerve-sparing robot-assisted radical cystoprostatectomy. Moreover, da Vinci system shortens the postoperative catheterization time from 14 days to only 7 days, allowing faster return to urinary continence from 160 days to 44 days, and quicker return time of erection from 440 days to 180 days. In addition the complication rate was lower in the robotic group (5\% vs. 20\%). (Menon et al.,2003)(Shah et al., 2014)

\section{Anderson-Hynes dismembered pyeloplasty and da Vinci}

The Anderson-Hynes pyeloplasty is a surgical method used to cure pelvi-ureteric junction obstruction. (Adam et al., 2015) There is an experiment performed to evaluate the use of da Vinci robotic system to perform laparoscopic Anderson- Hynes pyeloplasty. From June 2001 to February 2002, 9 patients performed laparoscopic AndersonHynes pyeloplasty with the da Vinci surgical system. The result depicts that the average blood loss was approximately 50mL less than normal laparoscopy in all cases with the average operative time of 138.8 minutes, and the mean suturing time was 62.4 minutes. The average length of hospitalization was 4.7 days. No intraoperative complications or open conversions were required. Additionally, all cases were successful within the average 4.1 month time period. Thus, the conclusion suggests that da Vinci assisted procedures provide a better result in overall operative times, suturing times, perioperative complications and success rate. (Matthew et al.,2002) 


\section{Analysis And Conclusion:-}

According to the survey I have conducted, $89.7 \%$ of people thought that robots had 2 legs and 2 arms like humans. When comparing robots to other living organisms, $87.2 \%$ think of humans. Although a lot more people believe that robotic surgical systems provide higher accuration, if they were to perform a high risk surgery, $69.2 \%$ prefer it to be done by a highly experienced surgeon while $30.8 \%$ will choose a robotic surgical system. This suggests that even though robots can perform better surgery, there is a trust that needs to be built in order for the population to adapt to the change that robots might replace surgeons in the future.

1. Adam, A., \& Smith, G. H. (2016). Anderson-Hynes pyeloplasty: are we all really on the same page?. ANZ journal of surgery, 86(3), 143-147. https://doi.org/10.1111/ans.13114

2. Bellis, M. (2019, July 3). Who Pioneered Robotics? ThoughtCo. https://www.thoughtco.com/timeline-ofrobots-1992363.

3. Farlex. (n.d.). nerve-sparing. The Free Dictionary. https://medical-dictionary.thefreedictionary.com/nervesparing.

4. Gettman, M. T., Neururer, R., Bartsch, G., \& Peschel, R. (2002). Anderson-Hynes dismembered pyeloplasty performed using the da Vinci robotic system. Urology, 60(3), 509-513. https://doi.org/10.1016/s00904295(02)01761-2

5. Hurley, S. (2017, March 3). Robot Surgery - the da Vinci Robot. Science Museum Blog. https://blog.sciencemuseum.org.uk/robot-surgery-the-da-vinci-robot/.

6. Inglis-Arkell, E. (2012, July 3). The very first robot "brains" were made of old alarm clocks. Gizmodo. https://gizmodo.com/the-very-first-robot-brains-were-made-of-old-alarm-cl-5890771.

7. Inventor of the Robot Arm and Its Continued Development. Collaborative robotic automation. (2019, October 1). https://www.universal-robots.com/blog/inventor-of-the-robot-arm-and-its-continued-development/.

8. Jimenez, L. (2014, November 6). 10 Amazing Robots Built Without Modern Technology. Listverse. https://listverse.com/2014/11/06/10-amazing-robots-built-without-modern-technology/.

9. Krishna, U. R., Sheth, S. S., \& Motashaw, N. D. (1979). Place of laparoscopy in pelvic inflammatory disease. Journal of obstetrics and gynaecology of India, 29(3), 505-510.

10. Mayo Foundation for Medical Education and Research. (2020, April 23). Pelvic inflammatory disease (PID). Mayo Clinic. https://www.mayoclinic.org/diseases-conditions/pelvic-inflammatory-disease/symptomscauses/syc-20352594.

11. Menon, M., Hemal, A. K., Tewari, A., Shrivastava, A., Shoma, A. M., El-Tabey, N. A., Shaaban, A., AbolEnein, H., \& Ghoneim, M. A. (2003). Nerve-sparing robot-assisted radical cystoprostatectomy and urinary diversion. BJU international, 92(3), 232-236. https://doi.org/10.1046/j.1464-410x.2003.04329.x

12. Navigation. Systems - I \& A. (n.d.). https://www.davincisurgerycommunity.com/systems_i_a.

13. NHS. (2018, August 1). NHS Choices. https://www.nhs.uk/conditions/laparoscopy/.

14. Shah, J., Vyas, A., \& Vyas, D. (2014). The History of Robotics in Surgical Specialties. American journal of robotic surgery, 1(1), 12-20. https://doi.org/10.1166/ajrs.2014.1006

15. Tanner, L. (2013, April 9). FDA takes fresh look at robotic surgery. USA Today. https://www.usatoday.com/story/news/nation/2013/04/09/robot-surgery-fda/2067629/.

16. Yu, H. Y., Hevelone, N. D., Lipsitz, S. R., Kowalczyk, K. J., \& Hu, J. C. (2012). Use, costs and comparative effectiveness of robotic assisted, laparoscopic and open urological surgery. The Journal of urology, 187(4), 1392-1398. https://doi.org/10.1016/j.juro.2011.11.089. 